J. Korean Math. Soc. 49 (2012), No. 5, pp. 965-976

http://dx.doi.org/10.4134/JKMS.2012.49.5.965

\title{
RICCI CURVATURE AND MONOPOLE CLASSES ON 3-MANIFOLDS
}

\author{
Chanyoung Sung
}

\begin{abstract}
We prove an $L^{2}$-estimate of Ricci curvature in terms of harmonic 1-forms on a closed oriented Riemannian 3-manifold admitting a solution of any rescaled Seiberg-Witten equations. We also give a necessary condition to be a monopole class on some special connected sums.
\end{abstract}

\section{Introduction}

A second cohomology class is called a monopole class if it arises as the first Chern class of a $\operatorname{Spin}^{c}$ structure for which the Seiberg-Witten equations admit a solution for every choice of a Riemannian metric. It is well-known by LeBrun $[9,10,12]$ that the existence of a monopole class gives various curvature estimates of a Riemannian 4-manifold. These immediately give corresponding estimates on 3-manifolds by using the dimensional reduction.

Theorem 1.1 ([19]). Let $(M, g)$ be a smooth closed oriented Riemannian 3manifold with $b_{1}(M) \geq 1$. Suppose that it admits a solution of the SeibergWitten equations for a Spin ${ }^{c}$ structure $\mathfrak{s}$. Then

$$
\int_{M}\left(s_{-}\right)_{g}^{2} d \mu_{g} \geq \frac{16 \pi^{2}\left|c_{1}(\mathfrak{s}) \cup[\omega]\right|^{2}}{\int_{M}|\omega|_{g}^{2} d \mu_{g}},
$$

where $\left(s_{-}\right)_{g}$ is $\min \left(s_{g}, 0\right)$ at each point and $s_{g}$ is the scalar curvature of $g$. Furthermore if the Seiberg-Witten invariant of $\mathfrak{s}$ is nonzero, then for a nonzero element $\omega$ in $H_{D R}^{1}(M)$

$$
\int_{M}\left|r_{g}\right|^{2} d \mu_{g} \geq \frac{8 \pi^{2}\left|c_{1}(\mathfrak{s}) \cup[\omega]\right|^{2}}{\int_{M}|\omega|_{g}^{2} d \mu_{g}},
$$

where $r_{g}$ is the Ricci curvature of $g$.

Received December 24, 2010.

2010 Mathematics Subject Classification. 57R57, 57M50, 53C99.

Key words and phrases. Seiberg-Witten equations, Ricci curvature, monopole class.

This work was supported by the National Research Foundation of Korea(NRF) grant funded by the Korea government(MEST) (No. 2011-0002791, 2012-0000341). 
Here, the Seiberg-Witten invariant in case of $b_{1}(M)=1$ means that of the chamber for arbitrarily small perturbations. We conjectured that the above Ricci curvature estimate still holds true when $c_{1}(\mathfrak{s})$ is a monopole class.

In this article, we show that it holds true if $c_{1}(\mathfrak{s})$ is a strong monopole class meaning that it is the first Chern class of a $\operatorname{Spin}^{c}$ structure of $M$ which admits a solution of the rescaled Seiberg-Witten equations of $\mathfrak{s}$ for any rescaling and any Riemannian metric (See Theorem 3.2).

Following LeBrun [13], we define the $f$-rescaled Seiberg-Witten equations on a 3-manifold $M$ to be

$$
\left\{\begin{array}{l}
D_{A} \Phi=0 \\
F_{A}=\left(\Phi \otimes \Phi^{*}-\frac{|\Phi|^{2}}{2} \mathrm{Id}\right) f,
\end{array}\right.
$$

where the rescaling factor $f$ is a positive smooth function on $M$. An obvious but important fact is that if the Seiberg-Witten invariant of $\mathfrak{s}$ is nonzero, then $c_{1}(\mathfrak{s})$ is a strong monopole class. But it is not known yet whether every monopole class is a strong monopole class.

In general, it is very difficult to find a monopole class which has zero SeibergWitten invariant. Connected sums of 4 -manifolds with $b_{2}^{+}>1$ are good candidates. In dimension 4, Bauer and Furuta $[2,1]$ devised a new refined invariant of Seiberg-Witten moduli space to prove the existence of a monopole class on some connected sums of Kähler surfaces. But it seems that no 3-dimensional example has been found yet. In the final section, we apply our curvature estimates to find a necessary condition to be a monopole class on some special connected sums.

For a brief introduction to the Seiberg-Witten theory, the readers are referred to $[16,20]$.

\section{The Yamabe problem for modified scalar curvature}

Let $(X, g)$ be a smooth closed oriented Riemannian 4-manifold and $W_{+}$be the self-dual Weyl curvature. By the modified scalar curvature we mean

$$
\mathfrak{S} \equiv s-\sqrt{6}\left|W_{+}\right|
$$

We will denote the set of $C^{2, \alpha}$ metrics for $\alpha \in(0,1)$ conformal to $g$ by $[g]$. Assume that there exists a metric in $[g]$ with nonpositive $\int_{X} \mathfrak{S} d \mu$. Then as observed by Gursky [6] and LeBrun [11], the standard proof of the Yamabe problem [14] proves that there exists a $C^{2, \alpha}$ metric in $[g]$ such that $\mathfrak{S}$ is a nonpositive constant. As in the Yamabe problem, it is a "minimizer" realizing

$$
\mathfrak{Y}(X,[g]) \equiv \inf _{\tilde{g} \in[g]} \frac{\int_{X} \mathfrak{S}_{\tilde{g}} d \mu_{\tilde{g}}}{\left(\operatorname{Vol}_{\tilde{g}}\right)^{\frac{1}{2}}} .
$$

We also have: 
Lemma 2.1. For $r \in[2, \infty]$,

$$
\mathfrak{Y}(X,[g])=-\inf _{\tilde{g} \in[g]}\left(\int_{X}\left|\mathfrak{S}_{\tilde{g}}\right|^{r} d \mu_{\tilde{g}}\right)^{\frac{1}{r}}\left(\operatorname{Vol}_{\tilde{g}}\right)^{\frac{1}{2}-\frac{1}{r}},
$$

where the infimum is realized only by the minimizer which is unique up to a constant multiplication.

Proof. The $L^{\infty}$ case is an immediate consequence of the other cases because of the inequality

$$
\left\|\mathfrak{S}_{\tilde{g}}\right\|_{L^{\infty}}\left(\operatorname{Vol}_{\tilde{g}}\right)^{\frac{1}{2}} \geq\left(\int_{X}\left|\mathfrak{S}_{\tilde{g}}\right|^{r} d \mu_{\tilde{g}}\right)^{\frac{1}{r}}\left(\operatorname{Vol}_{\tilde{g}}\right)^{\frac{1}{2}-\frac{1}{r}},
$$

whose equality is attained only when $\mathfrak{S}_{\tilde{g}}$ is constant.

For $r \in[2, \infty)$, we will use the technique of Besson, Courtois, and Gallot [3]. Let $g$ be a minimizer. Let $\tilde{g}=u^{2} g$, where $u: X \rightarrow \mathbb{R}^{+}$is a $C^{2}$ function. Note that $u$ satisfies the modified Yamabe equation

$$
\mathfrak{S}_{\tilde{g}} u^{3}=\mathfrak{S}_{g} u+6 \Delta_{g} u .
$$

Therefore

$$
\begin{aligned}
\left(\int_{X}\left|\mathfrak{S}_{\tilde{g}}\right|^{r} d \mu_{\tilde{g}}\right)^{\frac{1}{r}}\left(\operatorname{Vol}_{\tilde{g}}\right)^{\frac{1}{2}-\frac{1}{r}} & =\left(\int_{X}\left|\mathfrak{S}_{\tilde{g}}\right|^{r} u^{4} d \mu_{g}\right)^{\frac{1}{r}}\left(\int_{X} u^{4} d \mu_{g}\right)^{\frac{1}{2}-\frac{1}{r}} \\
& \geq \frac{\int_{X}-\mathfrak{S}_{\tilde{g}} u^{2} d \mu_{g}}{\left(\int_{X} d \mu_{g}\right)^{\frac{1}{2}}} \\
& =\frac{\int_{X}-\left(\mathfrak{S}_{g}+6 \frac{1}{u} d^{*} d u\right) d \mu_{g}}{\left(\operatorname{Vol}_{g}\right)^{\frac{1}{2}}} \\
& =\frac{\int_{X}\left(-\mathfrak{S}_{g}+6 \frac{|d u|^{2}}{u^{2}}\right) d \mu_{g}}{\left(\operatorname{Vol}_{g}\right)^{\frac{1}{2}}} \\
& \geq \frac{\int_{X}-\mathfrak{S}_{g} d \mu_{g}}{\left(\operatorname{Vol}_{g}\right)^{\frac{1}{2}}}
\end{aligned}
$$

where the first inequality is an application of the Hölder inequality, and the equality holds if and only if $u$ is a positive constant. It also follows that any minimizer is a constant multiple of $g$.

\section{Ricci curvature estimate}

Let us start with the following lemma:

Lemma 3.1. Let $(M, g)$ be a smooth closed oriented Riemannian 3-manifold and $\mathfrak{s}$ be a Spinc structure on it. If it admits a solution for a rescaled SeibergWitten equations, then any $C^{2, \alpha}$-metric $\tilde{g} \in[g]$ also has a solution of the rescaled Seiberg-Witten equations for $\mathfrak{s}$. 
Proof. We claim that if $(A, \Psi)$ is a solution with respect to $g$, then $\left(A, e^{-\varphi} \Psi\right)$ is a solution with respect to $\tilde{g}=e^{2 \varphi} g$. Mapping an orthonormal frame $\left\{e_{1}, e_{2}, e_{3}\right\}$ of $g$ to an orthonormal frame $\left\{e^{-\varphi} e_{1}, e^{-\varphi} e_{2}, e^{-\varphi} e_{3}\right\}$ of $\tilde{g}$ gives a global isomorphism of two orthonormal frame bundles and hence a global isometry of the Clifford bundles. Then the identity map between the spinor bundles is an isometry.

For a proof of the $\operatorname{Spin}^{c}$ Dirac equation, one is referred to [8], and the curvature equation is immediate from the fact that

$$
\left|F_{A}\right|_{\tilde{g}}=e^{-2 \varphi}\left|F_{A}\right|_{g}=e^{-2 \varphi} f|\Psi|_{g}^{2}=f\left|e^{-\varphi} \Psi\right|_{\tilde{g}}^{2},
$$

where $f$ is the rescaling factor.

Theorem 3.2. Let $(M, g)$ be a smooth closed oriented Riemannian 3-manifold with $b_{1}(M) \geq 1$ and $\mathfrak{s}$ be a Spinc structure on it. Suppose that it admits a solution for the rescaled Seiberg-Witten equations for any rescaling. Then for any smooth metric $\tilde{g}$ conformal to $g$ and any nonzero $\omega \in H_{D R}^{1}(M)$,

$$
\int_{M}\left|r_{\tilde{g}}\right|^{2} d \mu_{\tilde{g}} \geq \frac{8 \pi^{2}\left|c_{1}(\mathfrak{s}) \cup[\omega]\right|^{2}}{\int_{M}|\omega|_{\tilde{g}}^{2} d \mu_{\tilde{g}}},
$$

and the equality holds if and only if $(M, \tilde{g})$ is a Riemannian submersion onto $S^{1}$ with totally geodesic fiber isometric to a compact oriented surface of genus $\geq 1$ with a non-positive constant curvature metric whose volume form is a multiple of $* \omega$ for $\tilde{g}$-harmonic $\omega$, and $\left[c_{1}(\mathfrak{s})\right]$ is a multiple of $[* \omega]$ in $H_{D R}^{2}(M)$, where * denotes the Hodge star with respect to $\tilde{g}$.

Proof. In order to prove the inequality, we may assume $c_{1}(\mathfrak{s}) \neq 0 \in H^{2}(M, \mathbb{R})$. Then the Seiberg-Witten equations have an irreducible solution for any metric in $[g]$, implying that there cannot exist a metric in $[g]$ with nonnegative scalar curvature, and hence there exists a smooth metric in $[g]$ with negative scalar curvature.

For notational convenience, let $g$ be any smooth metric in $[g]$. Consider the product metric $g+d t^{2}$ on $M \times S^{1}$, where $t \in[0,1]$ is a global coordinate of $S^{1}$. By the previous section, there exists a $C^{2, \alpha}$ metric $\hat{g} \in\left[g+d t^{2}\right]$ which minimizes $\mathfrak{Y}\left(M \times S^{1},\left[g+d t^{2}\right]\right)$ satisfying that $s-\sqrt{6}\left|W_{+}\right|$is a negative constant.

Lemma 3.3. $\hat{g}$ is invariant under the translation along $S^{1}$-direction.

Proof. Let $\hat{g}=\left(g+d t^{2}\right) \psi$ for a positive smooth function $\psi$ on $M \times S^{1}$, and we will show $\psi(x, t)=\psi(x, t+c)$ for $(x, t) \in M \times S^{1}$ for any $c$.

Since $\left(g+d t^{2}\right) \psi(x, t+c)$ is also a minimizer, by Lemma 2.1 there exists a smooth positive function $\varphi$ on $S^{1}$ such that

$$
\psi(x, t+c)=\varphi(c) \psi(x, t)
$$

for any $(x, t)$. For any $c$,

$$
\int_{M \times S^{1}} \psi(x, t) d \mu_{g+d t^{2}}=\int_{M \times S^{1}} \psi(x, t+c) d \mu_{g+d t^{2}}
$$




$$
=\varphi(c) \int_{M \times S^{1}} \psi(x, t) d \mu_{g+d t^{2}},
$$

where the first equality is due to the translation invariance of $d t^{2}$. Since $\psi>0$, we conclude that $\varphi(c)=1$ for any $c$.

We write the metric $\hat{g}$ as the warped form $h+f^{2} d t^{2}$ for $f: M \rightarrow \mathbb{R}^{+}$where $h$ is the metric $f^{2} g$ on $M$. Let $\left\{e_{1}, e_{2}, e_{3}, e_{4}=\frac{\partial}{\partial t}\right\}$ be a local orthonormal frame $M \times S^{1}$ with respect to $h+d t^{2}$, and $\left\{\omega^{i} \mid i=1, \ldots, 4\right\}$ its dual coframe. Recall the first Cartan's structure equations:

$$
d \omega^{i}=-\omega_{j}^{i} \wedge \omega^{j},
$$

where $\omega_{j}^{i}$ are the connection 1-forms of $h+d t^{2}$. Obviously $\omega_{4}^{i}$ are all zero for all $i$. Take an orthonormal coframe of $\hat{g}$ as $\left\{\omega^{1}, \omega^{2}, \omega^{3}, f \omega^{4}\right\}$ and apply the first Cartan's structure equations to $\left\{\omega^{1}, \omega^{2}, \omega^{3}, f \omega^{4}\right\}$, then one can see that the connection 1 -forms $\hat{\omega}_{j}^{i}$ of $\hat{g}$ are given by

$$
\begin{gathered}
\hat{\omega}_{j}^{i}=\omega_{j}^{i} \quad \text { for } i, j=1,2,3, \\
\hat{\omega}_{j}^{4}=\frac{\partial f}{\partial e_{j}} \omega^{4} \quad \text { for } j=1,2,3 .
\end{gathered}
$$

Let $(A, \Phi)$ be a solution of the $\frac{1}{f}$-rescaled Seiberg-Witten equations for $\mathfrak{s}$ on $(M, h)$, whose existence is guaranteed by Lemma 3.1. Then it is a translationinvariant solution of the Seiberg-Witten equations for $\mathfrak{s}$ on $\left(M \times S^{1}, h+d t^{2}\right)$.

We claim that $\left(A, \frac{\Phi}{\sqrt{f}}\right)$ is a solution of the unrescaled Seiberg-Witten equations for $\mathfrak{s}$ on $\left(M \times S^{1}, h+f^{2} d t^{2}\right)$. Let's denote the objects of $\hat{g}=h+f^{2} d t^{2}$ corresponding to that of $h+d t^{2}$ by $\hat{\imath}$. The $\operatorname{Spin}^{c}$ Dirac equation reads

$$
\begin{aligned}
& \hat{D}_{A}\left(f^{-\frac{1}{2}} \Phi\right) \\
= & \sum_{i=1}^{4} \hat{e}_{i} \hat{\nabla}_{\hat{e}_{i}}\left(f^{-\frac{1}{2}} \Phi\right) \\
= & \sum_{i=1}^{4} \hat{e}_{i}\left(\frac{\partial}{\partial \hat{e}_{i}}\left(f^{-\frac{1}{2}} \Phi\right)+\frac{1}{2}\left(\sum_{j<k} \hat{\omega}_{j}^{k}\left(\hat{e}_{i}\right) \hat{e}_{j} \hat{e}_{k}+A\left(\hat{e}_{i}\right)\right) f^{-\frac{1}{2}} \Phi\right) \\
= & \sum_{i=1}^{3} e_{i}\left(\frac{\partial}{\partial e_{i}}\left(f^{-\frac{1}{2}} \Phi\right)+\frac{1}{2}\left(\sum_{j<k \leq 3} \omega_{j}^{k}\left(e_{i}\right) e_{j} e_{k}+A\left(e_{i}\right)\right) f^{-\frac{1}{2}} \Phi\right) \\
& +\frac{\hat{e}_{4}}{2}\left(\sum_{j=1}^{3} \frac{\partial f}{\partial e_{j}} \omega^{4}\left(\hat{e}_{4}\right) e_{j} \hat{e}_{4}\right) f^{-\frac{1}{2}} \Phi \\
= & \sum_{i=1}^{3} e_{i}\left(-\frac{f^{-\frac{3}{2}}}{2} \frac{\partial f}{\partial e_{i}} \Phi+f^{-\frac{1}{2}} \frac{\partial \Phi}{\partial e_{i}}+\frac{1}{2}\left(\sum_{j<k \leq 3} \omega_{j}^{k}\left(e_{i}\right) e_{j} e_{k}+A\left(e_{i}\right)\right) f^{-\frac{1}{2}} \Phi\right)
\end{aligned}
$$




$$
\begin{aligned}
& +\frac{1}{2}\left(\sum_{j=1}^{3} \frac{\partial f}{\partial e_{j}} \frac{1}{f} e_{j}\right) f^{-\frac{1}{2}} \Phi \\
= & f^{-\frac{1}{2}} D_{A} \Phi \\
= & 0,
\end{aligned}
$$

and the curvature equation reads

$$
\begin{aligned}
F_{A}^{\hat{+}} & =\frac{1}{2}\left(F_{A}+\left(*_{h} F_{A}\right) \wedge \hat{\omega}^{4}\right) \\
& \simeq \frac{1}{2}\left(F_{A}+\left(*_{h} F_{A}\right) \wedge \omega^{4}\right) \\
& =\frac{1}{f}\left(\Phi \otimes \Phi^{*}-\frac{|\Phi|^{2}}{2} \mathrm{Id}\right) \\
& =\left(f^{-\frac{1}{2}} \Phi\right) \otimes\left(f^{-\frac{1}{2}} \Phi\right)^{*}-\frac{\left|f^{-\frac{1}{2}} \Phi\right|^{2}}{2} \mathrm{Id},
\end{aligned}
$$

where the equivalence in the second line means the identification as an endomorphism of the plus spinor bundle (Mapping an orthonormal frame $\left\{e_{1}, \ldots, e_{4}\right\}$ of $h+d t^{2}$ to an orthonormal frame $\left\{e_{1}, \ldots, e_{3}, \hat{e}_{4}\right\}$ of $h+f^{2} d t^{2}$ gives a global isomorphism of two orthonormal frame bundles, inducing a global isometry of the Clifford bundles. Then the identity map between the spinor bundles is an isometry).

\section{Lemma 3.4.}

$$
\int_{M \times S^{1}}\left(\frac{2}{3} s_{g+d t^{2}}-2 \sqrt{\frac{2}{3}}\left|W_{+}\right|_{g+d t^{2}}\right)^{2} d \mu_{g+d t^{2}} \geq 32 \pi^{2}\left(\left(\pi^{*} c_{1}\right)^{+}\right)^{2},
$$

where $\left(\pi^{*} c_{1}\right)^{+}$is the self-dual harmonic part of $\pi^{*} c_{1}$ with respect to $g+d t^{2}$, and $\pi: M \times S^{1} \rightarrow M$ is the projection map.

Proof. This immediately follows from LeBrun's method of Theorem 2.2 in [11]. First by using Lemma 2.1,

$$
\begin{aligned}
& \int_{M \times S^{1}}\left(\frac{2}{3} s_{g+d t^{2}}-2 \sqrt{\frac{2}{3}}\left|W_{+}\right|_{g+d t^{2}}\right)^{2} d \mu_{g+d t^{2}} \\
\geq & \int_{M \times S^{1}}\left(\frac{2}{3} s_{\hat{g}}-2 \sqrt{\frac{2}{3}}\left|W_{+}\right| \hat{g}\right)^{2} d \mu_{\hat{g}},
\end{aligned}
$$

and the right hand side is equal to

$$
\left(\int_{M \times S^{1}} d \mu_{\hat{g}}\right)^{\frac{1}{3}}\left(\int_{M \times S^{1}}\left|\frac{2}{3} s_{\hat{g}}-2 \sqrt{\frac{2}{3}}\right| W_{+}|\hat{g}|^{3} d \mu_{\hat{g}}\right)^{\frac{2}{3}},
$$

because $\hat{g}$ has constant $\frac{2}{3} s-2 \sqrt{\frac{2}{3}}\left|W_{+}\right|$. Now we use the fact that $\left(M \times S^{1}, \hat{g}\right)$ admits a solution of the unrescaled Seiberg-Witten equations for $\mathfrak{s}$. Combining its Weitzenböck formula with the Weitzenböck formula for the self-dual harmonic 2 -forms, we conclude that (3.1) is greater than or equal to $32 \pi^{2}\left(\left(\pi^{*} c_{1}\right)^{+}\right)^{2}$. 
Now using the above lemma, we get

$$
\begin{aligned}
\int_{M}\left|r_{g}\right|^{2} d \mu_{g}= & \int_{M \times S^{1}}\left|r_{g+d t^{2}}\right|^{2} d \mu_{g+d t^{2}} \\
= & 8 \int_{M \times S^{1}}\left(\frac{s_{g+d t^{2}}^{2}}{24}+\frac{1}{2}\left|W_{+}\right|_{g+d t^{2}}^{2}\right) d \mu_{g+d t^{2}} \\
& -8 \pi^{2}(2 \chi+3 \tau)\left(M \times S^{1}\right) \\
\geq & \frac{1}{2} \int_{M \times S^{1}}\left(\frac{2}{3} s_{g+d t^{2}}-2 \sqrt{\left.\frac{2}{3}\left|W_{+}\right|_{g+d t^{2}}\right)^{2} d \mu_{g+d t^{2}-0}}\right. \\
\geq & 16 \pi^{2}\left(\left(\pi^{*} c_{1}\right)^{+}\right)^{2} \\
\geq & \frac{8 \pi^{2}\left|c_{1} \cup[\omega]\right|^{2}}{\int_{M}|\omega|_{g}^{2} d \mu_{g}}
\end{aligned}
$$

where the second equality is due to the 4-dimensional Chern-Gauss-Bonnet theorem, and the first inequality is simple applications of Hölder inequality which was proved in LeBrun [12].

Lemma 3.5. The equality of the theorem statement holds if and only if $(M \times$ $\left.S^{1}, g+d t^{2}\right)$ is a Kähler manifold of non-positive constant scalar curvature with the Kähler form a multiple of $* \omega+\omega \wedge d t$ for harmonic $\omega$, and $\left[c_{1}(\mathfrak{s})\right]$ is a multiple of $[* \omega]$ in $H_{D R}^{2}(M)$.

Proof. Let's first consider the case when $\left[c_{1}\right] \neq 0 \in H_{D R}^{2}(M)$. It is shown in [12] that both equalities in (3.2) and (3.3) hold if and only if $g+d t^{2}$ is a Kähler metric of negative constant scalar curvature with the Kähler form a multiple of $\left(\pi^{*} c_{1}\right)^{+}$. The equality in (3.4) holds if and only if

$$
\omega=\omega^{h}=* c_{1}^{h}
$$

where $(\cdot)^{h}$ denotes the $g$-harmonic part.

When $\left[c_{1}\right]=0 \in H_{D R}^{2}(M)$, the equality implies that the metric is Ricciflat (In fact, it's a flat manifold $T^{3} / \Gamma$, because the dimension is 3 ). By the Weitzenböck formula for 1 -forms, $\omega^{h}$ is a nonzero parallel 1 -form. Then $* \omega^{h}+\omega^{h} \wedge d t$ is a nonzero parallel 2-form on $\left(M \times S^{1}, g+d t^{2}\right)$, and hence a Kähler form with the obvious complex structure compatible with the orientation. Conversely suppose that $\left(M \times S^{1}, g+d t^{2}\right)$ is scalar-flat Kähler. Since a Kähler curvature is a (symmetric) section of $\wedge^{1,1} \otimes \wedge^{1,1}$, on any scalar-flat Kähler surface the Riemann curvature restricted to self-dual two forms must be zero, and hence so is $W_{+}$. Then by the 4-dimensional Chern-Gauss-Bonnet theorem,

$$
\begin{aligned}
\int_{M \times S^{1}}\left|r_{g+d t^{2}}\right|^{2} d \mu_{g+d t^{2}}= & \int_{M \times S^{1}}\left(\frac{1}{3}\left(s_{g+d t^{2}}\right)^{2}+4\left|W_{+}\right|_{g+d t^{2}}^{2}\right) d \mu_{g+d t^{2}} \\
& -8 \pi^{2}(2 \chi+3 \tau)\left(M \times S^{1}\right) \\
= & 0
\end{aligned}
$$


giving the equality.

If the equality holds, we have a parallel splitting of $T M$ by $\omega$ and $* \omega$, each of which gives a manifold of constant scalar curvature of dimension 1 and 2 respectively by the above lemma. Thus the universal cover of $(M, g)$ is isometric to $\mathbb{H}^{2} \times \mathbb{R}^{1}$ or $\mathbb{R}^{3}$. In the first case, $M$ is a quotient by a discrete subgroup of $\operatorname{PSL}(2, \mathbb{R}) \times \mathbb{Z}$, and in the second case, $M \times S^{1}$ is a complex torus or a bielliptic surface by the Enriques-Kodaira classification. Therefore $(M, g)$ is obtained by identifying two boundaries of $\Sigma \times[0,1]$ by an orientation-preserving isometry of a compact Riemann surface $\left(\Sigma, g_{c}\right)$ of genus $\geq 1$ with a constant curvature metric $g_{c}$. Then $(M, g)$ is locally a Riemannian product of $\left(\Sigma, g_{c}\right)$ and $S^{1}$, i.e., a Riemannian submersion onto $S^{1}$ with totally geodesic fiber $\left(\Sigma, g_{c}\right)$.

Conversely, suppose that $(M, g)$ is such an oriented Riemannian submersion $\pi: M \rightarrow S^{1}$. The metric being locally a product, $\left(M \times S^{1}, g+d t^{2}\right)$ is a Kähler manifold with an obvious complex structure and a Kähler form $d \Omega+\pi^{*} d s \wedge d t$ where $d \Omega$ is the volume form of $\left(\Sigma, g_{c}\right)$ and $d s$ is the volume form of the base. The scalar curvature of $g$ is just the constant scalar curvature of $g_{c}$ and the first Chern class of $\Sigma$ is a multiple of $d \Omega$ which is equal to $* \pi^{*} d s$. By the above lemma, $(M, g)$ attains the equality, thereby completing the proof.

Remark. As seen in the proof, the condition that $(M, g)$ admits a solution for the rescaled Seiberg-Witten equations for any rescaling is superfluous. It is enough to suppose that $\left(M \times S^{1}, \hat{g}\right)$ has a solution for the unrescaled SeibergWitten equations.

In particular, if $(M, g)$ has an isometric $G$-action which can be lifted to $\mathfrak{s}$ for a compact Lie group $G$, then it is enough for $M$ to have solutions of the Seiberg-Witten equations for any $G$-invariant metric. In this case, $c_{1}(\mathfrak{s})$ is called a $G$-monopole class, and a $G$-monopole class sometimes exists even when the ordinary Seiberg-Witten invariant vanishes. This will be dealt with in our forthcoming paper $([21])$.

Remark. It is well-known that on a compact Kähler surface $(X, g, \varpi)$ with $b_{2}^{+}(X)=1$, the Seiberg-Witten invariant of its canonical Spin ${ }^{c}$ structure for a small perturbation and a Riemannian metric $\tilde{g} \in[g]$ is equal to \pm 1 , if $\operatorname{deg}\left(c_{1}(X)\right):=\left[c_{1}(X)\right] \cdot[\varpi]$ is negative (See [16]), and also true for any perturbation and Riemannian metric if $c_{1}(X)$ is a torsion by using the celebrated Taubes's theorem [22] and the wall crossing formula [4, 15].

Now let's discuss some immediate implications of the above theorem. First, by taking $\omega$ to be $* c_{1}^{h}(\mathfrak{s})$ where $(\cdot)^{h}$ denotes the harmonic part, we have

$$
\int_{M}\left|r_{\tilde{g}}\right|^{2} d \mu_{\tilde{g}} \geq 8 \pi^{2} \int_{M}\left|c_{1}^{h}(\mathfrak{s})\right|_{\tilde{g}}^{2} d \mu_{\tilde{g}} .
$$

More interestingly we can get a lower bound of $L^{2}$-norm of a harmonic 1-form on $M$ : 
Corollary 3.6. Under the same hypothesis as Theorem 3.2, if $\tilde{g}$ is not flat,

$$
\left(\int_{M}|\omega|_{\tilde{g}}^{2} d \mu_{\tilde{g}}\right)^{\frac{1}{2}} \geq \frac{2 \sqrt{2} \pi|\alpha \cup[\omega]|}{\left(\int_{M}\left|r_{\tilde{g}}\right|^{2} d \mu_{\tilde{g}}\right)^{\frac{1}{2}}},
$$

where $\alpha$ is a convex combination of any two such $c_{1}(\mathfrak{s})$ 's.

\section{Monopole classes on connected sums}

Our curvature estimates provide an easy toolkit in the study of a closed 3manifold $M$ with a non-torsion monopole. In [19], we derived the inevitability of collapsing when such a manifold has zero Yamabe invariant which implies the existence of a sequence of unit-volume Riemannian metrics $\left\{g_{i}\right\}$ on $M$ satisfying $\inf _{i} \int_{M} s_{g_{i}}^{2} d \mu_{g_{i}}=0$. We also found a necessary condition to be a monopole class in a specific example as follows:

Proposition 4.1 ([19]). Let $M$ be a closed oriented 3-manifold which fibers over the circle with a periodic monodromy, and $N$ be a closed oriented 3manifold with $b_{1}(N)=0$. Then the rational part of a monopole class, if any, of $M \# N$ is of the form $m[F]$ for an integer $m$ satisfying $|m| \leq|\chi(F)|$, where $\chi(F)$ is the Euler characteristic of the fiber $F$.

(Warning: In the statement of Theorem 1.4 of [19], $b_{1}(N)=0$ is missing by mistake.)

We give a generalization of this to connected sums:

Proposition 4.2. Let $M_{i}$ for $i=1, \ldots, n$ be a closed oriented 3-manifold which fibers over the circle with a periodic monodromy, and $N$ be any closed oriented 3-manifold. Then the rational part of a monopole class, if any, of $M_{1} \# \cdots \# M_{n} \# N$ is of the form

$$
\beta+\sum_{i=1}^{n} m_{i}\left[F_{i}\right]
$$

for $\beta \in H^{2}(N, \mathbb{Z})$ and an integer $m_{i}$ satisfying $\left|m_{i}\right| \leq-\chi\left(F_{i}\right)$, where $\chi\left(F_{i}\right)$ is the Euler characteristic of the fiber $F_{i}$ in $M_{i}$.

Proof. Let $\alpha$ be a monopole class of $X=M_{1} \# \cdots \# M_{n} \# N$.

First if any $F_{i}$ is a 2-sphere, then the only possibility for $M_{i}$ is $S^{1} \times S^{2}$. Letting $[\omega]$ be the Poincaré-dual of $F_{i}$, we only have to show that it pairs zero with $\alpha$. Let $0<\varepsilon \ll 1$.

Take a metric of positive scalar curvature on the $M_{i}$. For the connected sum, we take a small ball on $M_{i}$, and a representative $\omega$ of $[\omega]$ to be supported outside of that ball. Then perform the Gromov-Lawson type surgery $[5,17,18]$ on it keeping the positivity of scalar curvature to get a compact manifold $M_{i}^{\prime}$ with a cylindrical end. And then contract it small enough so that

$$
\int_{M_{i}^{\prime}}|\omega|^{2} d \mu \leq \varepsilon
$$


On the other part of $X$, we put any metric such that it satisfies

$$
\int\left(s_{-}\right)^{2} d \mu<1
$$

and perform the Gromov-Lawson surgery such that the cylindrical end matches with that of the above-made $M_{i}^{\prime}$ while still satisfying (4.1).

After gluing these two pieces, we have that

$$
\int_{X}|\omega|^{2} d \mu \leq \varepsilon \quad \text { and } \quad \int_{X}\left(s_{-}\right)^{2} d \mu<1 .
$$

Applying Theorem 1.1, we get

$$
|4 \pi \alpha \cup[\omega]|^{2}<\varepsilon
$$

which proves $\alpha \cup[\omega]=0$.

Secondly, let's consider the case of $M_{i}$ with $\chi\left(F_{i}\right) \leq 0$. Let $[\omega] \in H^{1}\left(M_{i}, \mathbb{R}\right)$. By the Mayer-Vietoris principle, $H^{1}\left(M_{i}, \mathbb{R}\right)$ is generated by

$$
\pi_{i}^{*} d t, \quad \text { and } \quad\left\{[\sigma] \in H^{1}\left(F_{i}, \mathbb{R}\right) \mid f_{i}^{*}[\sigma]=[\sigma]\right\},
$$

where $\pi_{i}: M_{i} \rightarrow S^{1}$ is the projection map and $f_{i}$ is the monodromy diffeomorphism.

When $[\omega]$ is one of the latter ones, we have to show that it pairs zero with $\alpha$. We can express $\omega$ as

$$
\frac{1}{d_{i}} \sum_{n=1}^{d_{i}}\left(f_{i}^{n}\right)^{*} \sigma
$$

for such $\sigma$ satisfying $\left[f^{*} \sigma\right]=[\sigma]$, where $d_{i}$ is the order of $f_{i}$.

By taking a $f_{i}$-invariant metric on $F_{i}$, we can put a locally-product metric on $M_{i}$ such that $\pi_{i}$ is a Riemannian submersion with totally geodesic fibers onto a circle of radius $\varepsilon$ and

$$
\int\left(s_{-}\right)^{2} d \mu<\varepsilon .
$$

We can take a small simply-connected open set $B$ in $F_{i}$, which is invariant under $f_{i}$, and take a representative $\sigma$ of the above $[\sigma] \in H^{1}\left(F_{i}, \mathbb{R}\right)$ to be supported outside of $B$.

For the connected sum, we perform the Gromov-Lawson surgery on $B \times$ $I\left(\frac{\varepsilon}{10}\right) \subset M_{i}$ where $I\left(\frac{\varepsilon}{10}\right)$ is the interval of length $\frac{\varepsilon}{10}$ to get $M_{i}^{\prime}$ with a cylindrical end while still satisfying (4.2). On the other part of $X$, as before we put a metric with a cylindrical end isometric to that of this $M_{i}^{\prime}$ while satisfying (4.1).

After gluing two pieces, we have

$$
\int_{X}|\omega|^{2} d \mu \leq C \varepsilon \quad \text { and } \quad \int_{X}\left(s_{-}\right)^{2} d \mu<1+\varepsilon
$$

for a constant $C>0$. Hence by Theorem 1.1

$$
|4 \pi \alpha \cup[\omega]|^{2}<C \varepsilon(1+\varepsilon),
$$


proving $\alpha \cup[\omega]=0$.

Finally when $\omega=\pi_{i}^{*} d t$, the adjunction inequality on a 3 -manifold $X$, which can be proved in the same way as 4-manifolds ([7]) by considering $X \times S^{1}$ gives

$$
\left|\left\langle\alpha,\left[F_{i}\right]\right\rangle\right| \leq-\chi\left(F_{i}\right)
$$

completing the proof.

Remark. As noted, $M_{i} \times S^{1}$ for $M_{i}$ as above admits a Kähler metric of constant scalar curvature, and each $M_{i}$ admits a $d_{i}$-fold covering space which is $F_{i} \times S^{1}$.

For $M_{i}$ with $F_{i}=S^{2}$, one can use the argument of gluing of moduli spaces of Seiberg-Witten equations along cylindrical ends to prove $4 \pi \alpha \cup d t=0$.

It seems plausible to conjecture:

Conjecture 4.3. Let $N_{i}$ for $i=1, \ldots, n$ be a closed oriented 3-manifold. Then (the rational part of) any monopole class of $N_{1} \# \cdots \# N_{n}$ is expressed as $\sum_{i}^{n} \alpha_{i}$ where $\alpha_{i}$ is a monopole class of $N_{i}$.

Acknowledgement. The author would like to warmly thank Dr. Daewoong Chung for helpful discussions and providing a nice research environment at KIAS.

\section{References}

[1] S. Bauer, A stable cohomotopy refinement of Seiberg-Witten invariants. II, Invent. Math. 155 (2004), no. 1, 21-40.

[2] S. Bauer and M. Furuta, A stable cohomotopy refinement of Seiberg-Witten invariants. I, Invent. Math. 155 (2004), no. 1, 1-19.

[3] G. Besson, G. Courtois, and S. Gallot, Volume et entropie minimale des espaces localement symétriques, Invent. Math. 103 (1991), no. 2, 417-445.

[4] H.-D. Cao and J. Zhou, Equivariant cohomology and wall crossing formula in SeibergWitten theory, Math. Res. Lett. 5 (1998), no. 6, 711-721.

[5] M. Gromov and H. B. Lawson, The classification of simply connected manifolds of positive scalar curvature, Ann. of Math. 111 (1980), no. 3, 423-434.

[6] M. Gursky, Four-manifolds with $\delta W^{+}=0$ and Einstein constants of the sphere, Math. Ann. 318 (2000), no. 3, 417-431.

[7] P. B. Kronheimer and T. S. Mrowka, The genus of embedded surfaces in the projective plane, Math. Res. Lett. 1 (1994), no. 6, 797-808.

[8] H. B. Lawson and M.-L. Michelson, Spin Geometry, Princeton University Press, 1989.

[9] C. LeBrun, Four-manifolds without Einstein metric, Math. Res. Lett. 3 (1996), no. 2, $133-147$.

[10] Yamabe constants and the perturbed Seiberg-Witten equations, Comm. Anal. Geom. 5 (1997), no. 3, 535-553.

[11] _ Curvature and smooth topology in dimension four, Global analysis and harmonic analysis (Marseille-Luminy, 1999), 179-200, Semin. Congr., 4, Soc. Math. France, Paris, 2000

[12] _ Ricci curvature, minimal volumes, and Seiberg-Witten theory, Invent. Math. 145 (2001), no. 2, 279-316.

[13] _ Einstein metrics, four-manifolds, and differential topology, Surveys in differential geometry, Vol. VIII (Boston, MA, 2002), 235-255, Surv. Differ. Geom., VIII, Int. Press, Somerville, MA, 2003. 
[14] J. Lee and T. Parker, The Yamabe problem, Bull. Amer. Math. Soc. 17 (1987), no. 1, $37-91$.

[15] T. J. Li and A. Liu, General wall crossing formula, Math. Res. Lett. 2 (1995), no. 6, 797-810.

[16] J. Morgan, The Seiberg-Witten Equations and Applications to the Topology of Smooth Four-Manifold, Princeton University Press, 1996.

[17] C. Sung, Surgery, curvature, and minimal volume, Ann. Global Anal. Geom. 26 (2004), no. 3, 209-229.

[18] _ Surgery and equivariant Yamabe invariant, Differential Geom. Appl. 24 (2006), no. $3,271-287$.

[19] Collapsing and monopole classes of 3-manifolds, J. Geom. Phys. 57 (2007), no. $2,549-559$.

[20] _ Surgery, Yamabe invariant, and Seiberg-Witten theory, J. Geom. Phys. 59 (2009), no. 2, 246-255.

[21] , Finite group actions and G-monopole classes on smooth 4-manifolds, arXiv: 1108.3875

[22] C. H. Taubes, The Seiberg-Witten invariants and symplectic forms, Math. Res. Lett. 1 (1994), no. 6, 809-822.

Department of Mathematics and Institute for Mathematical Sciences KONKUK UNIVERSITY

SEOUl 143-701, Korea

E-mail address: cysung@kias.re.kr 